\title{
ACUTE RETROGRADE AORTIC DISSECTION DURING OPERATIONS FOR RUPTURED STANFORD TYPE B DISSECTION
}

Kazuhito Imanaka, MD, Shunei Kyo, MD, Hiroaki Tanabe, MD, Hiroshi Ohuchi, MD, Haruhiko Asano, MD, and Yuji Yokote, MD, Saitama, Japan

In general, resection of an intimal tear is desirable during operations for aortic dissection. In patients with rupture of acute Stanford type B dissection, however, aggressive resection of an intimal tear sometimes appears to be too dangerous, especially when the tear is in the vicinity of the aortic arch. Emergency replacement of the ruptured segment only, which is mandatory for saving the patient, can be a method of choice. We report a pitfall in such conservative operations.

Clinical summary. A 54-year-old hypertensive man had a sudden onset of severe back pain and paraplegia and was transferred to our hospital. On admission, his blood pressure was $60 \mathrm{~mm} \mathrm{Hg}$ and both lower limbs were completely paralyzed. Computed tomography of the chest and transesophageal echocardiography (TEE) revealed Stanford type B aortic dissection, a large amount of thrombus in the pseudolumen, a possible but unclear main intimal tear in the distal aortic arch, hematoma around the descending aorta, and left pleural effusion. There was no dissection in the ascending aorta or the transverse arch, and pericardial effusion was absent. Pleuracentesis yielded bloody pleural effusion. Rupture of the dissected descending aorta was diagnosed, and an emergency operation was performed. Through a left posterolateral thoracotomy, with the use of a femoro-femoral cardiopulmonary bypass, both proximal and distal portions of the descending aorta were gently clamped with the heart beating, and about a $20-\mathrm{cm}$ length of the descending aorta was successfully replaced with a prosthetic graft. Although the patient was in profound shock before the operation and repeatedly required the injection of vasopressor agents, his postoperative hemodynamic condition was stabilized. However, repeat TEE during the wound closure disclosed dissection in the ascending aorta and the aortic arch and a considerable amount of

From the First Department of Surgery, Saitama Medical School, Saitama, Japan.

J Thorac Cardiovasc Surg 2001;121:1215-6

Copyright () 2001 by The American Association for Thoracic Surgery

0022-5223/2001 $\$ 35.00+0 \quad \mathbf{1 2 / 5 4 / 1 1 1 6 5 3}$

doi: $10.1067 / \mathrm{mtc} .2001 .111653$ pericardial effusion, which had been absent preoperatively. The tear was present in the distal aortic arch. Intraoperative retrograde aortic dissection and cardiac tamponade were diagnosed. The chest wound was quickly closed, and then the patient underwent replacement of the ascending aorta through a midline sternotomy. The dissection affected the entire aorta above the coronary artery ostia, and the pericardial cavity was filled with blood. The second operation was also carried out uneventfully. Perhaps because of his preoperative moribund state and the large scale of the operation, the patient convalesced rather slowly. Two months later, during his prolonged hospital stay, he suddenly died of an aortoesophageal fistula at the proximal anastomosis of the prosthetic graft in the descending aorta, possibly as a result of graft infection.

Discussion. Currently, patients with acute Stanford type B aortic dissection are usually given medical treatment, and surgical intervention is indicated only if rupture or organ ischemia occurs. In such cases, whether the intimal tear should be treated is a matter of controversy. ${ }^{1,2}$ When the intimal tear is near the aortic arch, its complete resection often requires an open proximal anastomosis under deep hypothermic circulatory arrest. Even though this method is theoretically desirable, it requires considerable circumspection before it can be applied to any patient because the patient's general condition is often very poor. We believe that a limited operation should be considered, at least for those in a state of shock. Because in our patient the site of the main intimal tear was unclear and the patient was in profound shock, we did not aim to resect the intimal tear. We followed a strategy of limited surgical repair but encountered another unexpected pitfall.

The clinical prognosis of acute retrograde dissection of the ascending aorta is not favorable. ${ }^{3,4}$ Among our 34 patients with this condition, 28 were surgically treated, resulting in a hospital mortality of $35 \%$ (including the present case), which is higher than that found with the other type of acute Stanford type A dissection. It should be kept in mind that there are increasing hazards that will cause retrograde dissection of the ascending aorta to develop during limited operations for acute Stanford type B dissection. The dissected aorta has to be clamped, which may cause another tear, even when the pseudolumen is thrombosed. Moreover, especially in patients 
in a state of shock, vasopressor agents are often administered repeatedly or continuously. When the aorta is clamped with the heart beating under partial cardiopulmonary bypass, it is not always possible, even with cautious management, to control the blood pressure, which can suddenly become prohibitively high. Moreover, full heparinization may have played some role in the extended progression of the retrograde dissection of the ascending aorta and acute cardiac tamponade, which together may have caused unexpected circulatory collapse. When the ruptured segment is excluded by the placement of clamps, surgeons tend to feel secure. However, the potentially fatal pitfall still exists.

Also, the importance of intraoperative TEE monitoring must be reemphasized. ${ }^{5}$ We did not notice the development of retrograde dissection of the ascending aorta until TEE was repeated at the end of the procedure. In some cases a delayed diagnosis can have a fatal outcome. Another striking aspect is that without TEE the development of ascending aortic dissection in an anesthetized patient may be unable to be diagnosed until serious consequences occur. The procedure for the rupture of the descending aorta by itself may not require intraoperative TEE. However, it is indispensable in view of every possible problem.

All of the above factors can be avoided if the intimal tear is resected. However, we believe at present that our strategy of using a limited operation for moribund patients with acute Stanford type B dissection is justified. When a limited operation is performed, however, we must be aware of the possible hazards of the development of retrograde dissection of the ascending aorta, either during or after the operation. We should observe and manage such cases more carefully than usual cases. Even though the patient's condition appears to be stable, examinations including either TEE or transthoracic echocardiography and computed tomography should be performed meticulously.

Received for publication Sept 8, 2000; accepted for publication Sept 14, 2000.

Address for reprints: Kazuhito Imanaka, MD, First Department of Surgery, Saitama Medical School, 38 Morohongo, Moroyama-machi, Iruma-gun, Saitama 3500495, Japan (E-mail: imanaka@ saitama-med.ac.jp).

\section{REFERENCES}

1. Yun KL, Glower DD, Miller DC, Fann JI, Mitchell RS, White $\mathrm{WD}$, et al. Aortic dissection resulting from tear of transverse arch: Is concomitant arch repair warranted? J Thorac Cardiovasc Surg 1991;102:355-70.

2. Guilmet DG, Bachet J, Goudot B, Dreyfus G, Martinelli GL. Aortic dissection: anatomic types and surgical approaches. J Cardiovasc Surg 1993;34:23-32.

3. Ebel R, Oelert H, Meyer J, Puth M, Mohr-Katory S, Hausmann $\mathrm{D}$, et al. Effect of medical and surgical therapy on aortic dissection evaluated by transesophageal echocardiography-implications for prognosis and therapy. Circulation 1993;87:1604-15.

4. von Segesser LK, Killer I, Ziswiler M, Linka A, Ritter M, Jenni $\mathrm{R}$, et al. Dissection of the descending thoracic aorta extending into the ascending aorta: a therapeutic challenge. $J$ Thorac Cardiovasc Surg 1994;108:755-61.

5. Kyo S, Takamoto S, Omoto R, Matsumura M, Kimura S, Neya K, et al. Intraoperative echocardiography for diagnosis and treatment of aortic dissection-utility of color flow mapping for surgical decision making in acute stage. Herz 1992;17:377-89. 